hep-ph/0109219

RM3-TH 01/11

\title{
Highlights of Short Baseline Physics at a Neutrino Factory
}

\author{
Stefano Forte ${ }^{1}$ \\ INFN, Sezione di Roma III, via della Vasca Navale 84, I-00146 Roma, Italy
}

\begin{abstract}
We review the physics potential for experiments with intense neutrino beams at the front-end of a muon storage ring, stressing the way the spin and flavour structure of neutrino interactions with matter can be used to shed light on the structure of the strong and electroweak interactions. Specifically, we discuss precision tests of the standard model, studies of polarized and unpolarized structure functions of the nucleon, and several new and exotic physics items.
\end{abstract}

Invited plenary talk at NuFact $\mathbf{0 1}$

Tsukuba, Japan, May 2001

to be published in the proceedings

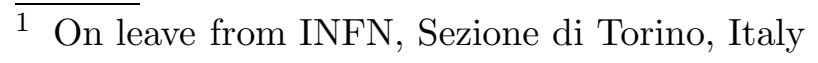




\section{Probing Matter with Intense Neutrino Beams}

Front-end physics at a neutrino factory is based on the realization that, because of the flavour and spin structure of the coupling of neutrinos to weak currents, a neutrino beam is a unique probe of the structure of the standard model and of the structure of the nucleon. A neutrino beam thus has a greater physics potential than conventional electron or muon beams, provided the intensity of the beam is high enough. An accurate assessment of the physics potential of the experiments which could be performed with an intense neutrino beam cannot abstract from the fact that the time scale for the construction of a neutrino factory is of order of ten years: clearly, it is difficult to envisage what the 'standard model' will be ten years from now. Here, we will discuss the physics case for these experiments based on present-day knowledge. This is interesting not only because many of the measurements that we will discuss would only be possible at a neutrino factory (for instance, those related to polarized parton distributions), but also because comparison with what we already know will allow us to highlight the peculiar features of physics with neutrino beams.

This brief review is largely based on a recent detailed quantitative study performed by a CERN working group [1]; quantitative estimates given here are taken from there unless otherwise stated. Previous studies on the physics potential of neutrino factories have been performed by working groups at Fermilab [2] and Brookhaven [3]. General background on neutrino experiments is in Ref. [4]. Even though we will usually describe the energy and luminosity dependence of our results, we will assume the 'CERN reference scenario' [5]: specifically, a $50 \mathrm{GeV} \mu$ beam, with $10^{20}$ muon decays per year along a $100 \mathrm{~m}$ straight section.

\section{Neutrino interactions with matter}

The most interesting neutrino-induced reactions in matter are neutrino-electron elastic scattering, and neutrino-nucleon (deep)-inelastic scattering (DIS), i.e. neutrino-quark scattering. The former is a clean purely weak interaction process, while the latter allows one to probe strongly-interacting matter with weak currents.

The cross section for elastic neutrino- or antineutrino-electron scattering is

$$
\frac{d \sigma}{d y}=\frac{2 G_{F}^{2} m_{e} E_{\nu}}{\pi}\left[g_{L}^{2}+g_{R}^{2}(1-y)^{2}\right]
$$


where $y \equiv E_{e} / E_{\nu}, 0 \leq y \leq 1$, so the total cross section is obtained replacing $(1-y)^{2} \rightarrow \frac{1}{3}$. The couplings for neutral-current (NC) processes are $g_{L}=\frac{1}{2}\left(g_{V}+\lambda_{\nu} g_{A}\right), g_{R}=\frac{1}{2}\left(g_{V}-\lambda_{\nu} g_{A}\right)$, with $\lambda_{\nu}=-1\left(\lambda_{\bar{\nu}}=1\right)$, and for charged-current (CC) processes $g_{L}=1\left(g_{L}=0\right), g_{R}=0\left(g_{R}=1\right)$ for neutrinos (antineutrinos). The total couplings are listed in the following table (the numerical values are computed with $\left.\sin ^{2} \theta_{W}=0.23\right)$ :

\begin{tabular}{|c|c|c|c|}
\hline Reaction & $g_{L}$ & $g_{R}$ & $g_{L}^{2}+\frac{1}{3} g_{R}^{2}$ \\
\hline$\nu_{\mu} e^{-} \rightarrow \nu_{\mu} e^{-}(\mathrm{NC})$ & $-\frac{1}{2}+\sin ^{2} \theta_{W}$ & $\sin ^{2} \theta_{W}$ & 0.091 \\
$\overline{\nu_{\mu}} e^{-} \rightarrow \overline{\nu_{\mu}} e^{-}(\mathrm{NC})$ & $\sin ^{2} \theta_{W}$ & $-\frac{1}{2}+\sin ^{2} \theta_{W}$ & 0.077 \\
$\nu_{e} e^{-} \rightarrow \nu_{e} e^{-}(\mathrm{NC}+\mathrm{CC})$ & $\frac{1}{2}+\sin ^{2} \theta_{W}$ & $\sin ^{2} \theta_{W}$ & 0.551 \\
$\overline{\nu_{e}} e^{-} \rightarrow \overline{\nu_{e}} e^{-}(\mathrm{NC}+\mathrm{CC})$ & $\sin ^{2} \theta_{W}$ & $\frac{1}{2}+\sin ^{2} \theta_{W}$ & 0.231 \\
\hline
\end{tabular}

The total cross-section is tiny, of order $\sigma \sim 10^{-3} \times\left(g_{L}^{2}+\frac{1}{3} g_{R}^{2}\right)$ pb for $50 \mathrm{GeV}$ neutrinos. Yet at a neutrino factory with a 20 ton liquid argon TPC or a fully active 2 ton liquid methane target one expects integrated luminosities of order of $8.6 \cdot 10^{10} \mathrm{pb}^{-1}$, leading to rates of order of $\sim 10^{7}$ events per year with a $\mu^{+}$ beam (half with a $\mu^{-}$beam).

The neutrino-nucleon DIS cross section is by a factor $\sim m_{p} / m_{e}$ larger: for charged-current interactions, up to corrections suppressed by powers of $m_{p}^{2} / Q^{2}$

$$
\begin{aligned}
& \frac{d^{2} \sigma^{\lambda_{p} \lambda_{\ell}}\left(x, y, Q^{2}\right)}{d x d y}=\frac{G_{F}^{2}}{2 \pi\left(1+Q^{2} / m_{W}^{2}\right)^{2}} \frac{Q^{2}}{x y}\left\{\left[-\lambda_{\ell} y\left(1-\frac{y}{2}\right) x F_{3}\left(x, Q^{2}\right)\right.\right. \\
& \left.+(1-y) F_{2}\left(x, Q^{2}\right)+y^{2} x F_{1}\left(x, Q^{2}\right)\right]-2 \lambda_{p}\left[-\lambda_{\ell} y(2-y) x g_{1}\left(x, Q^{2}\right)\right. \\
& \left.\left.-(1-y) g_{4}\left(x, Q^{2}\right)-y^{2} x g_{5}\left(x, Q^{2}\right)\right]\right\}
\end{aligned}
$$

where $\lambda$ are the lepton and proton helicities (assuming longitudinal proton polarization), and the kinematic variables are $y=\frac{p \cdot q}{p \cdot k}$ (lepton fractional energy loss), $x=\frac{Q^{2}}{2 p \cdot q}$ (Bjorken $x$ ). The neutral-current cross-section is found from eq. (2) by letting $m_{W} \rightarrow m_{Z}$ and multiplying by an overall factor $\left[\frac{1}{2}\left(g_{V}-\right.\right.$ $\left.\left.\lambda_{\ell} g_{A}\right)\right]^{2}$.

At a neutrino factory, structure functions could be measured for $0.01 \leq$ $x \leq 1$ and $1 \leq Q^{2} \leq 100 \mathrm{GeV}^{2}$ (note the kinematic limit $s \equiv \frac{Q^{2}}{x y} \leq$ $\left.2 m_{p} E_{\mu}=100 \mathrm{GeV}^{2}\right)$. Because $y=Q^{2} /\left(2 x m_{p} E_{\nu}\right)$, at fixed $x$ and $Q^{2}, y$ only varies with the neutrino energy. At a neutrino factory with a broad-band beam, neutrinos of various energies (measurable on an event-by-event basis) are available. It is then possible to disentangle all structure functions $F_{i}$ (unpolarized) or $g_{i}$ (polarized) by fitting the $y$ dependence of the data for 
fixed $x$ and $Q^{2}$. One expects statistical errors of order of $1 \%$ or better on all three structure functions for $x \gtrsim 0.1$ when $Q^{2} \lesssim 15 \mathrm{GeV}^{2}$, and for $x \gtrsim 0.3$ when $Q^{2} \lesssim 80 \mathrm{GeV}^{2}$, and of order $10 \%$ otherwise.

Information on the structure of the nucleon target is encoded in the structure functions, whose leading parton content in terms of the unpolarized and polarized quark distribution for the $i$-th flavor $q_{i} \equiv q_{i}^{\uparrow \uparrow}+q_{i}^{\uparrow \downarrow}$ and $\Delta q_{i} \equiv q_{i}^{\uparrow \uparrow}-q_{i}^{\uparrow \downarrow}$ is summarized in the following table, where for comparison we also give the standard result for charged-lepton scattering via virtual photon exchange:

$$
\begin{array}{ccc}
\mathrm{NC} & F_{1}^{\gamma}=\frac{1}{2} \sum_{i} e_{i}^{2}\left(q_{i}+\bar{q}_{i}\right) & g_{1}^{\gamma}=\frac{1}{2} \sum_{i} e_{i}^{2}\left(\Delta q_{i}+\Delta \bar{q}_{i}\right) \\
\mathrm{NC} & F_{1}^{Z}=\frac{1}{2} \sum_{i}\left(g_{V}^{2}+g_{A}^{2}\right)_{i}\left(q_{i}+\bar{q}_{i}\right) & g_{1}^{Z}=\frac{1}{2} \sum_{i}\left(g_{V}^{2}+g_{A}^{2}\right)_{i}\left(\Delta q_{i}+\Delta \bar{q}_{i}\right) \\
\mathrm{NC} & F_{3}^{Z}=2 \sum_{i}\left(g_{V} g_{A}\right)_{i}\left(q_{i}+\bar{q}_{i}\right) & g_{1}^{Z}=-\sum_{i}\left(g_{V} g_{A}\right)_{i}\left(\Delta q_{i}+\Delta \bar{q}_{i}\right) \\
\mathrm{CC} & F_{1}^{W^{+}}=\bar{u}+d+s+\bar{c} & g_{1}^{W^{+}}=\Delta \bar{u}+\Delta d+\Delta s+\Delta \bar{c} \\
\mathrm{CC} & -F_{3}^{W^{+}} / 2=\bar{u}-d-s+\bar{c} & g_{5}^{W^{+}}=\Delta \bar{u}-\Delta d-\Delta s+\Delta \bar{c} \\
& F_{2}=2 x F_{1} & g_{4}=2 x g_{5}
\end{array}
$$

Here $e_{i}$ are the electric charges and $\left(g_{V}\right)_{i},\left(g_{A}\right)_{i}$ are the weak charges of the $i$-th quark flavour. If $W^{+} \rightarrow W^{-}$(incoming $\bar{\nu}$ beam), then $u \leftrightarrow d, c \leftrightarrow s$. The structure functions $F_{3}, g_{4}$ and $g_{5}$ are parity-violating, and therefore not accessible in virtual photon scattering. Of course, beyond leading order in the strong coupling each quark or antiquark flavor's contribution receives $O\left(\alpha_{s}\right)$ corrections proportional to itself and to all other quark, antiquark and gluon distributions. This last correction is flavor-blind, and thus decouples from the parity-violating structure functions $F_{3}, g_{4}$ and $g_{5}$.

\section{Tests of the Standard Model}

\subsection{The weak mixing angle}

The weak mixing angle can be determined from the leptonic weak couplings $g_{L}$ and $g_{R}$, which can be cleanly extracted from the measurement of the elastic cross-section eq. (1). The main background for this process is quasielastic neutrino-nucleon scattering. Because the transverse momentum of the outgo-

ing electron is $p_{t} \sim \sqrt{m_{e} E_{\nu}}$, but $p_{t} \sim \sqrt{m_{p} E_{\nu}}$ for scattering off nucleons, the background can be removed with a $p_{t}$ cut. The current best determination from this process is

$$
\sin ^{2} \theta_{W}=0.2324 \pm 0.0058 \text { (stat) } \pm 0.0059 \text { (syst) }
$$


from the CHARMII experiment [6].
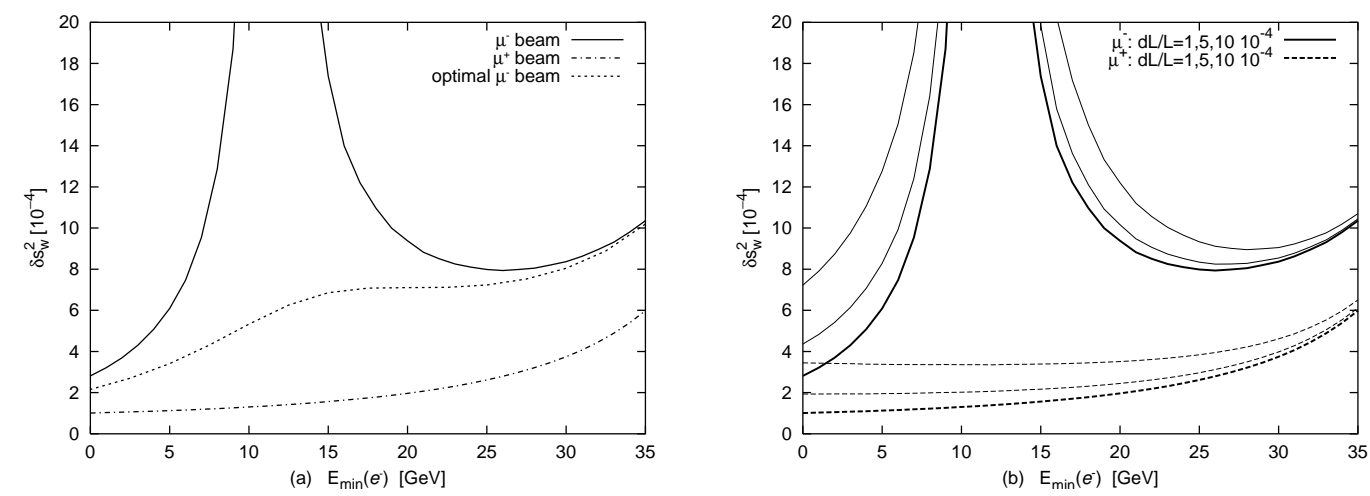

Fig. 1. (a) Statistical uncertainty (in units $10^{-4}$ ) in the extraction of $\sin ^{2} \theta_{W}$ from $\nu e$ scattering as a function of the minimum electron energy. (b) Impact of luminosity measurement at the level of $10^{-3}, 510^{-4}, 1 \cdot 10^{-4}$ on the same $\sin ^{2} \theta_{W}$ sensitivities.

At a neutrino factory, events without a muon in the final state originating from $\nu_{\mu}$ or $\bar{\nu}_{e}$ (in a $\mu^{-}$beam) cannot be distinguished and must be considered together. With the luminosity discussed above, one then gets the statistical accuracy shown in Fig. 1a. The main systematic uncertainty is the determination of the incoming neutrino flux (see Fig. 1b), which can be done by normalizing to the inverse muon decay, namely the process $\bar{\nu}_{e} e^{-} \rightarrow \mu^{-} \bar{\nu}_{\mu}$ or $\nu_{\mu} e^{-} \rightarrow \mu^{-} \nu_{e}$

An independent determination of $\sin ^{2} \theta_{W}$ can be obtained from DIS. This has the advantage of larger statistics, but the disadvantage of systematic uncertainties related to knowledge of the nucleon structure. The value of $\sin ^{2} \theta_{W}$ is extracted from the quark weak couplings: in ratios of NC to CC DIS crosssections eq. (2) the lepton couplings cancel. Also, such ratios are less sensitive to details of nucleon structure, in that the leading-order dependence on parton distributions can be made to cancel. In particular, the Paschos-Wolfenstein [7] ratio

$$
R^{-}=\frac{\sigma_{N C}\left(\nu_{\mu}\right)-\sigma_{N C}\left(\bar{\nu}_{\mu}\right)}{\sigma_{C C}\left(\nu_{\mu}\right)-\sigma_{C C}\left(\bar{\nu}_{\mu}\right)}=\frac{1}{2}-\sin ^{2} \theta_{W}
$$

has been used to obtain a determination [8]

$$
\sin ^{2} \theta_{W}(\mathrm{OS})=0.2255 \pm 0.0018 \text { (stat) } \pm 0.0010 \text { (syst) }
$$

At a neutrino factory, one can only measure combinations where $\nu_{\mu}$ and $\bar{\nu}_{e}$ do not have to be disentangled on an event-by-event basis, such as $R^{\mu^{-}}=$ $\frac{\sigma_{N C}\left(\nu_{\mu}\right)+\sigma_{N C}\left(\bar{\nu}_{e}\right)}{\sigma_{C C}\left(\nu_{\mu}\right)+\sigma_{C C}\left(\bar{\nu}_{e}\right)}, R^{\mu^{+}}=\frac{\sigma_{N C}\left(\bar{\nu}_{\mu}\right)+\sigma_{N C}\left(\nu_{e}\right)}{\sigma_{C C}\left(\bar{\nu}_{\mu}\right)+\sigma_{C C}\left(\nu_{e}\right)}$, or $P=\frac{\sigma_{N C}\left(\mu^{-}\right)-\sigma_{N C}\left(\mu^{+}\right)}{\sigma_{C C}\left(\mu^{-}\right)-\sigma_{C C}\left(\mu^{+}\right)}$, or linear combination thereof. These combinations give a good handle on $\sin ^{2} \theta_{W}$, but are more dependent on nucleon structure: in fact, it is convenient to construct 
an optimal combination, which maximizes the dependence on $\sin ^{2} \theta_{W}$ while minimizing the uncertainty due to parton distributions. The uncertainties on $\sin ^{2} \theta_{W}$ in units of $10^{-4}$ for various combinations are given in the following table:

\begin{tabular}{|c|c|c|}
\hline observable & stat. error & PDF \\
\hline$R^{\mu^{-}}$ & 0.4 & $\sim 12$ \\
\hline$R^{\mu^{+}}$ & 0.5 & $\sim 15$ \\
\hline$R^{\mu^{-}}-0.8 R^{\mu^{+}}$ & 2.2 & $\sim 2$ \\
\hline$P$ & 4.9 & $\sim 4$ \\
\hline
\end{tabular}

In summary, at a neutrino factory the weak mixing angle could be measured to an accuracy of about $10^{-4}$, in two different ways. This is comparable to the best presently available determination of $\sin ^{2} \theta_{W}$, namely [9] $\sin ^{2} \theta_{W}=0.23098 \pm 0.00026$ from all available asymmetry measurements. Furthermore, the two measurements from neutrino elastic scattering and DIS undergo different radiative corrections from each other and from the asymmetry measurements. This would therefore be a very competitive test of the standard model, or its violation.

\subsection{The strong coupling}

The strong coupling can be extracted from DIS data either by considering combinations which do not depend on parton structure (sum rules), or by performing global fits where both $\alpha_{s}$ and all parton distributions are simultaneously determined. In both cases neutrino beams are superior to conventional charged lepton beams because of the availability of more independent combinations of individual parton distributions.

Specifically, if both proton and neutron targets are available, one may construct two combinations which only depend on the total number of up plus down valence quarks (Gross-Lewellyn Smith sum rule [10]) or up minus down valence quarks (unpolarized Bjorken sum rule [11]), up to an $\alpha_{s}$-dependent factor which is currently known to $O\left(\alpha_{s}^{3}\right)$ :

$$
\begin{aligned}
& S_{\mathrm{GLS}}^{N}\left(Q^{2}\right)=\frac{1}{2} \int_{0}^{1} d x\left(F_{3}^{\nu p}\left(x, Q^{2}\right)+F_{3}^{\nu n}\left(x, Q^{2}\right)\right) \\
& \quad=C_{\mathrm{GLS}}\left(Q^{2}\right) \int_{0}^{1} d x\left[u\left(x, Q^{2}\right)-\bar{u}\left(x, Q^{2}\right)+d\left(x, Q^{2}\right)-\bar{d}\left(x, Q^{2}\right)\right]
\end{aligned}
$$




$$
\begin{aligned}
& \quad=3\left(1-\frac{\alpha_{s}\left(Q^{2}\right)}{\pi}-3.25\left[\frac{\alpha_{s}\left(Q^{2}\right)}{\pi}\right]^{2}-12.2\left[\frac{\alpha_{s}\left(Q^{2}\right)}{\pi}\right]^{3}+\ldots\right)+\frac{h_{\mathrm{GLS}}}{Q^{2}} \\
& S_{\mathrm{BjU}}^{N}\left(Q^{2}\right)=\frac{1}{2} \int_{0}^{1} d x\left(F_{1}^{\nu p}\left(x, Q^{2}\right)-F_{1}^{\nu n}\left(x, Q^{2}\right)\right) \\
& \quad=C_{\mathrm{BjU}}\left(Q^{2}\right) \int_{0}^{1} d x\left[u\left(x, Q^{2}\right)-\bar{u}\left(x, Q^{2}\right)-\left(d\left(x, Q^{2}\right)-\bar{d}\left(x, Q^{2}\right)\right)\right] \\
& \quad=1\left(1-\frac{2}{3} \frac{\alpha_{s}\left(Q^{2}\right)}{\pi}-2.65\left[\frac{\alpha_{s}\left(Q^{2}\right)}{\pi}\right]^{2}-13.38\left[\frac{\alpha_{s}\left(Q^{2}\right)}{\pi}\right]^{3}+\ldots\right)+\frac{h_{\mathrm{BjU}}}{Q^{2}},
\end{aligned}
$$

where the coefficients of the power corrections $h_{\mathrm{GLS}}$ and $h_{\mathrm{BjU}}$ are unknown and must be fitted. The disadvantage of this way of determining $\alpha_{s}$ is the need to extrapolate over the full range $0 \leq x \leq 1$ the data which are only available in a limited range of $x$. The accessible $x$ range is larger at lower $Q^{2}$, where however power suppressed corrections are larger. At present, a determination of $\alpha_{s}\left(M_{Z}\right)$ with an error $\Delta \alpha_{s}\left(M_{Z}\right)={ }_{-0.012}^{+0.009}$ has been obtained from the GLS sum rule with the CCFR neutrino beam [12], while the BjU integral has never been measured because of the impossibility of disentangling $F_{1}$ and $F_{2}$ from present-day neutrino DIS data. At the neutrino factory, one could reach an uncertainty $\Delta \alpha_{s}\left(M_{Z}\right)=0.0035$, which is completely dominated by limited kinematic coverage in either $Q^{2}$ (power corrections) or $x$ (small $x$ extrapolation) and could only be improved if a higher energy beam were available.

A very competitive determination could be obtained by performing a global fit to structure functions: interestingly, because more structure functions are available, at a neutrino factory the full set of parton distributions could be determined in a single experiment (see Sect. 4 below). One could then achieve a statistical accuracy on $\alpha_{s}$ of order $\Delta \alpha_{s}\left(M_{Z}\right)=0.0003$, to be compared to the statistical accuracy $\Delta \alpha_{s}\left(M_{Z}\right) \approx 0.0017$ which can be obtained from presentday global fits [13] which include both $\nu$ and charged-lepton DIS data from various experiments (the error from current neutrino experiments alone is of order $\left.\Delta \alpha_{s}\left(M_{Z}\right) \approx 0.005[14]\right)$. The statistical precision of this determination of $\alpha_{s}$ value is by one order of magnitude better than the extant global error [15] $\Delta \alpha_{s}\left(M_{Z}\right) \sim 0.003$. The accuracy in the determination of $\alpha_{s}$ at a neutrino factory would thus be entirely dominated by theoretical uncertainties, and it could be the most accurate determination once next-to-next-to-leading order corrections to perturbative evolution [16] are known.

More studies of strong interaction physics could take advantage of the fact that charm is copiously produces in charged-current events and easily detected. This could be exploited not only in order to further refine our knowledge of parton distributions through the study of specific semi-inclusive channels, but also to study QCD corrections to charm production near threshold, and 
finally to measure accurately absolute branching ratios and decay constants of individual charmed mesons, such as $\Lambda_{c}$ or $D_{s}$.

\section{The Structure of the Nucleon}

Current information on the parton distributions of the nucleon [17] comes mostly from DIS data, the bulk of which are produced with charged lepton beams and thus are essentially NC scattering. The problem is then that (see Sect. 2) only one combination of $q_{i}+\bar{q}_{i}$ distributions is accessible. This means that different quark flavors can only be disentangled using isospin, if proton and neutron targets are available, plus in principle by exploiting perturbative evolution (i.e. subleading corrections). Hence, using NC DIS data it is hard to determine strangeness, and impossible to measure the $\mathrm{C}$-odd combination $q-\bar{q}$.

This is to be contrasted with the situation in CC scattering [18], where if both proton and neutron targets and $\nu$ and $\bar{\nu}$ beams are available, then one can form eight linear combinations of the two independent structure functions ( $F_{1}$ and $F_{3}$ unpolarized, $g_{1}$ and $g_{5}$ polarized), six of which are independent (NC data do not give any extra independent information). It is easy to construct leading-order combinations of parton distributions which, below charm threshold, determine all six light flavours and anti-flavours independently. The charm distribution can then be determined either comparing data below and above charm threshold, or tagging charm events, which have a distinct dimuon signature [4]. In practice, of course, parton distributions will be determined by fitting the full next-to-leading order expression of structure functions, however the fact that individual partons can already be disentangled at a leading-order guarantees the accuracy of the NLO determination.

\subsection{Unpolarized DIS: the flavor content of the nucleon}

Unpolarized parton distributions are a necessary ingredient in the computation of any collider process. However, only the up, down and gluon distributions can be determined in a reasonably accurate way from DIS data. Some information on strangeness can be extracted [19] from neutrino data, while some less-inclusive observables (such as $W$ production, or Drell-Yan) provide some constraints on the relative size of the $q$ and $\bar{q}$ distributions, but the results are at best semi-quantitative (see Fig. 2a).

A quantitative estimate of the accuracy at a neutrino factory can be ob-

tained by generating pseudo-data for structure functions with appropriate 

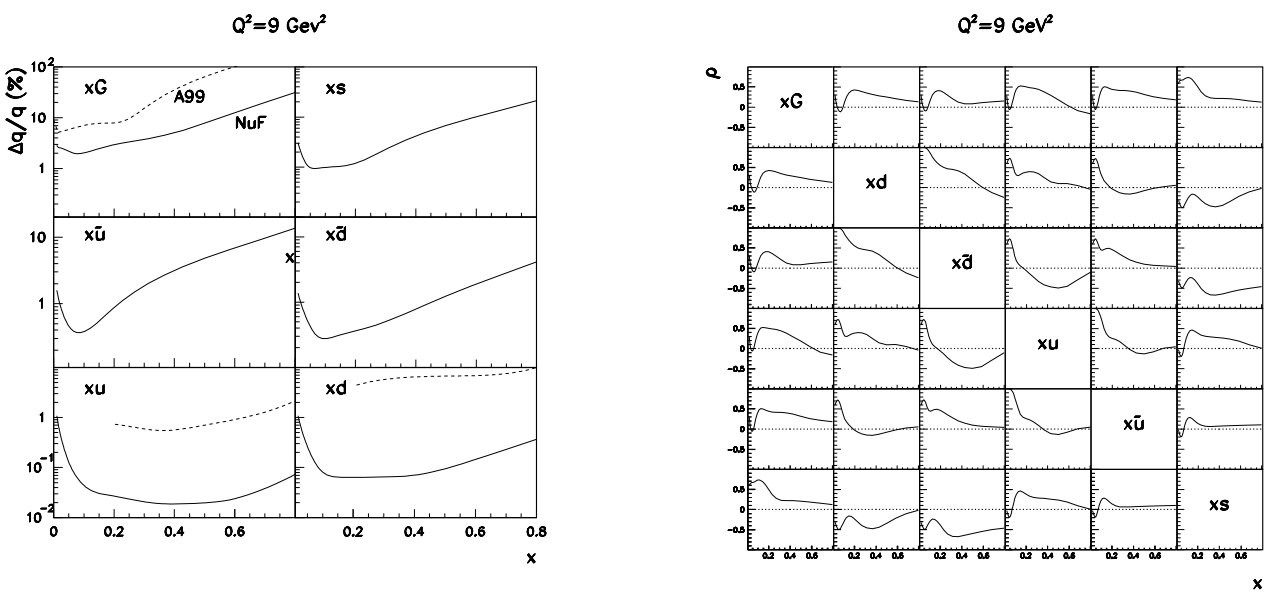

Fig. 2. (a) Percentage error on parton distributions at a neutrino factory (solid) compared with present-day [13] errors (dashed). (b) Correlation coefficients between parton distributions determined at a neutrino factory.

errors, and then producing a fit of parton distributions based on this data. In Fig. 2a the error estimates on individual partons obtained in this way are compared to the extant knowledge. Note that no current errors on strange and antiquark distributions are given, since the present results largely depend on theoretical prejudice. In Fig. $2 \mathrm{~b}$ we further show that the point-by-point correlation of individual distributions is uniformly quite low, indicating that a model-independent flavour and antiflavour separation is possible to $10 \%-20 \%$ accuracy in most of the accessible kinematic range. A neutrino factory would essentially turn the determination of individual parton distributions into a precision quantitative exercise.

\subsection{Polarized DIS: the spin of the nucleon}

Polarized DIS has recently attracted considerable attention because the unexpected smallness of the proton's singlet axial charge $a_{0}$ suggests that the nucleon spin structure is considerably subtler than naive parton expectations might suggest [20]. In the naive parton model the singlet axial charge is the fraction of the nucleon spin which is carried by quarks; the Zweig rule would lead one to expect this to be around $60 \%$ but the experimental value is compatible with zero.

This state of affairs point to several possible scenarios for the nucleon spin structure. Beyond leading order the axial charge is given by

$$
a_{0}=\Delta \Sigma-\frac{n_{f} \alpha_{s}}{2 \pi} \Delta G
$$


where $\Delta \Sigma=\sum_{i}\left(\Delta q_{i}+\Delta \bar{q}_{i}\right)$ is the scale-invariant quark spin fraction, and $\Delta G$ is the gluon spin fraction. The latter, due to the axial anomaly, gives an effectively leading-order contribution to $a_{0} 7$ (it depends on scale as $\Delta G \sim \frac{1}{\alpha_{s}}$ ). So a first possibility is that $\Delta G$ is large enough that the quark spin $\Delta \Sigma$ is large even though the axial charge $a_{0}$ is small ('anomaly' scenario [21]). A different option ('instanton' scenario) is that $\Delta \Sigma$ is itself small, because of a large contribution from sea quarks whose polarization is anticorrelated to that of valence quarks (possibly because of 'instanton' $Q C D$ vacuum configurations [22]). Yet another possibility ('skyrmion' scenario) is that $\Delta \Sigma$ is small because of a large contribution from 'valence' strange quarks $|\Delta s|>>|\Delta \bar{s}|$ (as suggested [23] in the Skyrme model).

At present, the quark and gluon spin fractions can be extracted from NC DIS data [24]: $\Delta G\left(1,1 \mathrm{GeV}^{2}\right)=0.8 \pm 0.2, \Delta \Sigma(1)=0.38 \pm 0.03$, while the 'octet' combination can be determined using $\mathrm{SU}(3)$ from baryon $\beta$-decay constants: $a_{8} \equiv \Delta u+\Delta d-2 \Delta s=0.6 \pm 30 \%$ (the large error comes from a conservative estimate of $\mathrm{SU}(3)$ violation). It is of course impossible to polarize the kind of targets which are required for present-day neutrino DIS experiments, so no neutrino data are available, and thus little information on strangeness and no information at all on the quark-antiquark separation is available in the polarized case. At a neutrino factory, significant rates could be achieved with small targets [18]: with a detector radius of $50 \mathrm{~cm}, 100 \mathrm{~m}$ length, the structure functions $g_{1}, g_{5}$ could be independently measured to an accuracy which is about one order of magnitude better than that with which $g_{1}$ is determined in present charged lepton DIS experiments.

We can then assess the information that one might obtain on the nucleon spin structure by generating pseudodata within different representative scenarios [25]. Assuming an 'anomaly' scenario one would determine $\Delta g=0.9 \pm 0.1$; $\Delta \Sigma=0.39 \pm 0.01 ; a_{8}=0.56 \pm 0.01$, while in an 'instanton' scenario $\Delta g=$ $0.2 \pm 0.1 ; \Delta \Sigma=0.32 \pm 0.01 ; a_{8}=0.57 \pm 0.01$. Furthermore, in an 'instanton' scenario $[\Delta s-\Delta \bar{s}]\left(1,1 \mathrm{GeV}^{2}\right)=-0.007 \pm 0.007$; while in a "skyrmion" scenario one would observe $[\Delta s-\Delta \bar{s}]\left(1,1 \mathrm{GeV}^{2}\right)=-0.106 \pm 0.008$. Note that the strange contribution, and hence the octet component, would be determined from the data directly, without having to use SU(3) (which would thus be tested in the process). Clearly, the distinct scenarios could be well separated from each other: in fact full flavor separation at the level of first moments would be possible. Hence, a resolution of the nucleon spin structure would be possible at a neutrino factory.

The situation would be less favorable for the $x$-dependence of parton distributions, because of the difficulty of disentangling the potentially large gluon contribution. If the gluon were well determined from other experiments, however, a full point-by-point determination of the polarized parton content of the nucleon would be possible, to an accuracy comparable to that of the un- 
polarized case.

\section{Exotica}

On top of the standard processes discussed so far, the high luminosity at a neutrino factory allows the study of several rare or exotic processes. of which we give two examples.
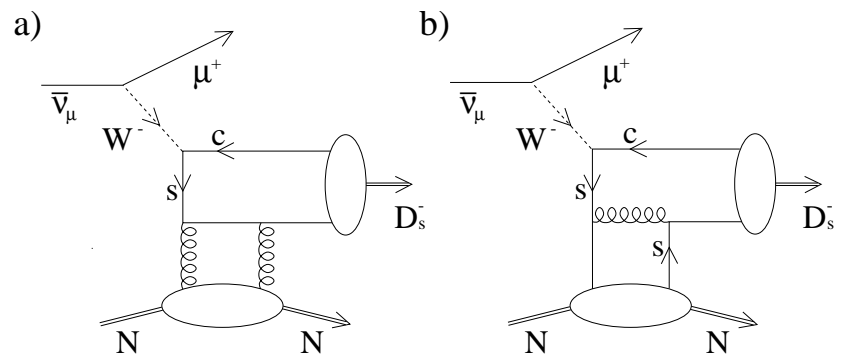

Fig. 3. Feynman diagram for hard exclusive $D_{s}$ production

A first example is the extraction of off-diagonal (or skewed) parton distributions (SPD) from hard exclusive meson production. The Feynman diagrams for this process are shown in Fig. 3. If the virtuality $Q^{2}$ of the $W$ boson is large compared to the nucleon momentum transfer $t$ and all masses, then the cross section for the process factorizes [26] as $\frac{d \sigma}{d x_{B j} d Q^{2} d t}\left(W^{-}+N \rightarrow D_{s}^{-}+N\right)=$ $H \otimes \Phi_{D} \otimes F$, where $H$ is the cross-section for the underlying hard perturbative parton subprocess, $\Phi_{D}$ is the $D^{-}$fragmentation function, and $F\left(x, t, Q^{2}\right)$ is an SPD, which interpolates between the usual nucleon form factor $G(t)$ (which is related to the first $x$-moment of $F$ ) and parton distribution $F(x)$ (related to the $t \rightarrow 0$ limit of $F$ ). The estimated cross section for this process is $\sigma=2.2 \times 10^{-5} \mathrm{pb}$, leading to $10^{4}$ event/yr at the neutrino factory. Given good knowledge of the $D_{s}$ fragmentation, the SPD can be measured. Because of relatively low backgrounds and the distinct experimental signature, this would be a favourable way of measuring SPDs, which have never been determined so far.

As another interesting 'exotic' process, consider neutrino-electron annihilation into hadrons through radiation of an intermediate virtual $W$ [3]. One can then define the analogue of $R$-ratio of $e^{+} e-$ annihilation:

$$
R_{A} \equiv \frac{\sigma\left(\bar{\nu}_{e} e^{-} \rightarrow \text { hadrons }\right)}{\sigma\left(\bar{\nu}_{e} e^{-} \rightarrow \bar{\nu}_{\mu} \mu^{-}\right)}
$$

$R_{A}(s)$ is the spectral function for annihilation into axial vector final states: at large $\sqrt{s}, R_{A}(s)$ can be computed in perturbative QCD from the underlying parton processes, while at low $\sqrt{s} \approx m_{\pi}, R_{A}(s)$ can be determined using 
PCAC from the relevant axial current matrix element. The threshold energy for the inverse muon decay process $\left(\bar{\nu}_{\mu} \mu^{-}\right.$final state) is $E_{\nu}=11 \mathrm{GeV}$, while the threshold for the lowest hadronic final state $\gamma \pi^{-}$is $E_{\nu}=19 \mathrm{GeV}$, so at a neutrino factory one could perform some interesting tests of PCAC and chiral symmetry.

\section{New Physics}

All the standard physics items which we discussed so far can also be viewed as tests for new physics, or tools in searches for new physics. Specifically, different determinations of the weak mixing angle lead to tests of the electroweak sector through the comparison of the corresponding radiative corrections (Sect. 3.1). Determinations of the strong coupling (Sect. 3.2) and especially its running test the strongly interacting sector. Precision determinations of parton distributions (Sect. 4) are a necessary input in searches for new physics: in fact some recent possible indications of new physics could also be explained by invoking lack of accurate knowledge of parton distributions [27].

However, high-intensity neutrino beams can also be used to design specific searches for new physics, of which we also give two examples. First, one can exploit the copious production of charmed mesons to study specific nonstandard decay channels. An interesting possibility is the search for possible $\mathrm{T}$-violation in the decays of the $\Lambda_{c}$ [3]. Indeed, in the semileptonic decay $\Lambda_{c}^{+} \rightarrow l^{+} \nu \Lambda$ one can construct an experimentally observable T-odd correlation $C_{T} \equiv\left\langle\vec{\sigma}_{\Lambda} \cdot\left(\vec{p}_{\Lambda} \times \vec{p}_{l}\right)\right\rangle$ from the spin and momentum of the daughter hyperon and the momentum of the final-state lepton. This correlation can-

not be affected by either strong or electromagnetic final state interactions, so a nonzero value for it is a measure of $\mathrm{T}$-violation.

Another example is the search for lepton-flavour violating decays, such as $\mu \rightarrow e \bar{\nu}_{\tau} \nu_{i}$. The corresponding four-Fermi couplings could be probed at a neutrino factory down to a strength about $\sim 3 \times 10^{-4}$ weaker than ordinary weak interactions. Of course stronger bounds already exist in the charged lepton sector, but the current limits for processes involving neutrinos are only of order $\sim 10^{-1}-10^{-2}$.

\section{Outlook}

The peculiar features of a neutrino beam is the availability of a probe which depends both on spin and flavor. However, the physics potential of such a beam can only be exploited given high enough intensity. Consequently, there 
is a whole class of measurements which is only possible at a neutrino factory. An example is charged-current polarized deep-inelastic scattering, which can only be studied accurately with a neutrino beam and a reasonably sized target: with charged lepton beams it is hard to separate charged-current and neutralcurrent events, and with low intensities it is impossible to polarize the target. Which of these measurements might be the most interesting at a neutrino factory will largely depend on the development of high-energy physics in the next decade. In any case, a neutrino factory has the potential of developing a broad program of short-baseline physics, which could answer many outstanding questions which are not likely to be addressed at any other experimental facility.

Acknowledgements: I thank Y. Kuno for inviting me to this very stimulating meeting, A. Donini for several discussions during the conference, and R. Ball and G. Ridolfi for a critical reading of the manuscript.

\section{References}

[1] M. L. Mangano et al., hep-ph/0105155.

[2] C. Albright et al., hep-ex/0008064.

[3] I. I. Bigi et al., hep-ph/0106177.

[4] J. M. Conrad, M. H. Shaevitz and T. Bolton, Rev. Mod. Phys. 70 (1998) 1341.

[5] http://muonstoragerings.web.cern.ch/muonstoragerings/; see also R. Garoby, these proceedings, CERN-PS-2001-055-RF.

[6] P. Vilain et al. [CHARM-II Collaboration], Phys. Lett. B 335 (1994) 246.

[7] E. A. Paschos and L. Wolfenstein, Phys. Rev. D 7 (1973) 91.

[8] K. S. McFarland et al. [NuTeV Collaboration], hep-ex/9806013.

[9] See e.g. E. Tournefier, hep-ex/0105091.

[10] D. J. Gross and C. H. Llewellyn Smith, Nucl. Phys. B14 (1969) 337.

[11] J. D. Bjorken, Phys. Rev. 163 (1967) 1767.

[12] J. H. Kim et al., Phys. Rev. Lett. 81 (1998) 3595.

[13] S. I. Alekhin, Phys. Rev. D 63 (2001) 094022.

[14] S. I. Alekhin and A. L. Kataev, Phys. Lett. B 452 (1999) 402.

[15] S. Bethke, J. Phys. G G26 (2000) R27.

[16] S. Moch, J. A. Vermaseren and M. Zhou, hep-ph/0108033. 
[17] H. L. Lai et al. [CTEQ Collaboration], Eur. Phys. J. C12, 375 (2000). A. D. Martin, R. G. Roberts, W. J. Stirling and R. S. Thorne, Eur. Phys. J. C 4 (1998) 463; for a review see e.g. S. Forte, Nucl. Phys. A 666 (2000) 113.

[18] R. D. Ball, D. A. Harris and K. S. McFarland, hep-ph/0009223.

[19] V. Barone, C. Pascaud and F. Zomer, Eur. Phys. J. C12, 243 (2000).

[20] See e.g. S. Forte, hep-ph/9409416; R. D. Ball and H. A. Tallini, J. Phys. G G25 (1999) 1327; G. M. Shore, hep-ph/9812355.

[21] G. Altarelli and G. G. Ross, Phys. Lett. B212 (1988) 391; R. D. Carlitz, J. C. Collins and A. H. Mueller, Phys. Lett. B214 (1988) 229.

[22] S. Forte, Phys. Lett. B224 (1989) 189; Nucl. Phys. B331 (1990) 1; S. Forte and E. V. Shuryak, Nucl. Phys. B357 (1991) 153.

[23] S. J. Brodsky, J. Ellis and M. Karliner, Phys. Lett. B206 (1988) 309; J. Ellis and M. Karliner, hep-ph/9601280.

[24] G. Altarelli, R.D. Ball, S. Forte and G. Ridolfi, Nucl. Phys. B496 (1997) 337; G. Altarelli, R. D. Ball, S. Forte and G. Ridolfi, Acta Phys. Pol. B29 (1998) 1145 .

[25] S. Forte, M. L. Mangano and G. Ridolfi, Nucl. Phys. B602 (2001) 585.

[26] J. C. Collins, L. Frankfurt and M. Strikman, Phys. Rev. D 56 (1997) 2982.

[27] S. Kuhlmann, H. L. Lai and W. K. Tung, Phys. Lett. B 409 (1997) 271. 